\title{
GERENCIAMENTO DOS RESÍDUOS EM UNIDADES DE SAÚDE EM MUNICÍPIOS DO ESTADO DE GOIÁS, BRASIL
} WASTE MANAGEMENT IN HEALTH UNITS IN MUNICIPALITIES IN THE STATE OF GOIAS, BRAZIL

\section{Poliana Nascimento Arruda}

Doutoranda em Ciências Ambientais na Universidade Federal de Goiás (UFG) - Goiás (GO), Brasil.

\section{Aline Souza Carvalho Lima}

Mestre em Engenharia do Meio Ambiente pela UFG -Goiás (GO), Brasil.

\section{Karla Alcione da Silva Cruvinel}

Professora assistente na Escola de Engenharia Civil e Ambiental da UFG - Goiás (GO), Brasil.

\section{Paulo Sérgio Scalize}

Professor associado na Escola de Engenharia Civil e Ambiental da UFG - Goiás (GO), Brasil.

\section{Endereço para correspondência:} Paulo Sérgio Scalize Avenida Universitária, 1.488 Setor Universitário - 74605-220 Goiânia (GO), Brasil E-mail: pscalize.ufg@gmail.com

Recebido: 29/07/2015

Aceito: $21 / 03 / 2017$

\section{RESUMO}

A presente pesquisa objetivou analisar o gerenciamento dos resíduos de serviços de saúde (RSS) em unidades de saúde públicas municipais em 17 municípios do estado de Goiás. Foram aplicados formulários aos gestores dos hospitais e postos de saúde, baseados em normas específicas para RSS. O levantamento das informações ocorreu no período de fevereiro de 2013 a janeiro de 2014, e verificou-se que a incineração é o método de tratamento utilizado total ou parcialmente em $100 \%$ dos municípios, sendo que $11 \%$ lançam parte de seus resíduos no mesmo local destinado aos resíduos domiciliares. Nota-se que os profissionais envolvidos no processo de gerenciamento dos RSS não possuem conhecimento sobre o mesmo em sua totalidade, refletindo em falhas na segregação e, consequentemente, nos custos de tratamento e disposição final, sendo necessária maior atenção do Poder Público e dos gestores para a questão dos RSS, promovendo a saúde dos trabalhadores e a qualidade ambiental do município.

Palavras-chave: resíduos de serviços de saúde; gestão municipal; segregação.

\section{ABSTRACT}

The objective of this work was to analyze the management of health care waste (HCW) in municipal public health facilities in 17 municipalities in the state of Goiás, Brazil. Forms have been applied to managers of hospitals and clinics based on specific standards for HCW. The survey of information occurred from February 2013 to January 2014, and it was found that incineration is the main method of treatment used in $100 \%$ of the municipalities, and $11 \%$ disposed part of the waste in the same place of the household waste. It was noticed that professionals involved in the HCW management processes have no knowledge of it at all, reflecting on failure on segregation and, therefore, on the treatment and disposal costs, being necessary higher attention of the public authorities and managers to the issue of $\mathrm{HCW}$, promoting health of workers and the environmental quality of the city.

Keywords: health care waste; municipal management; segregation. 


\section{INTRODUÇÃO}

A problemática da produção excessiva de resíduos sólidos, causada pelo desenvolvimento econômico e tecnológico, aliada ao crescimento populacional é um dos maiores desafios da atualidade, sendo necessárias ações para o seu gerenciamento correto por meio de técnicas empregadas desde a geração até a disposição final, sendo primordial quando se trata de resíduos perigosos.

No Brasil, entre os anos de 2011 e 2012 houve um aumento de $1,3 \%$ na geração de resíduos sólidos urbanos (RSU), contudo, a abrangência de sua coleta também sofreu um crescimento de $1,9 \%$. Porém, em relação à destinação final, 24 milhões de toneladas de RSU ainda são encaminhados para lixões e aterros controlados, não havendo diferença significativa em relação ao ano anterior (ABRELPE, 2012).

Além dos RSU, os resíduos de serviços de saúde (RSS) merecem significativa atenção devido ao seu potencial de contaminação, apesar de seu volume ser relativamente baixo, representando de 1 a 3\% do montante de RSU, e sua má gestão acarreta riscos aos trabalhadores dos estabelecimentos de saúde, à população e ao meio ambiente (ABRELPE, 2012; ROCHA, 2012).

Diante do Panorama de Resíduos Sólidos no Brasil, a Associação Brasileira de Empresas de Limpeza Pública e Resíduos Especiais (ABRELPE) concluiu que dos 5.565 municípios estudados em 2012, 4.282 municípios prestaram, total ou parcialmente, serviços relativos ao manejo dos RSS, levando a um índice médio de $1,5 \mathrm{~kg} \cdot \mathrm{hab}^{-1}$ por ano, resultando em um crescimento de $3 \%$ no total coletado em relação ao ano anterior (ABRELPE, 2012). Nota-se que o aumento da geração dos RSS foi superior ao dos RSU devido à crescente abrangência dos serviços de saúde oferecidos à população nesses últimos anos, resultando em serviços mais especializados com simultânea ampliação da oferta de materiais descartáveis no ambiente hospitalar, objetivando a segurança dos pacientes. Sendo assim, o manejo correto é de substancial importância para um ambiente limpo e saudável, colaborando com a segurança dos trabalhadores e do ambiente (PEREIRA, 2012).

Segundo PÉPIN et al. (2014), o descarte incorreto de seringas utilizadas nos serviços de saúde oferece riscos aos profissionais de saúde, da limpeza, além de criar oportunidades de reutilização desses materiais, sendo essa última prática responsável por cerca de 33.877 novas infecções por HIV, 1,7 milhões de infecções por hepatite B e 315.120 por hepatite C, em 2010. Portanto, o cumprimento de todas as etapas do gerenciamento, a destinação de recursos e o desenvolvimento de novas tecnologias para o tratamento e descarte corretos e seguros dos RSS devem ser parte integrante do sistema de saúde do país.

Devido a importância do manuseio adequado e o potencial crescimento da geração desses resíduos, foram criadas no Brasil legislações para nortear a gestão por meio de ações que visam à segurança e qualidade ambiental. Em 2004, a Agência Nacional de Vigilância Sanitária (ANVISA) implantou a RDC no 306 (ANVISA, 2004), que estabelece normas e responsabiliza os geradores pela gestão de seus resíduos, desde a coleta até a disposição final, por meio da implantação do Plano de Gerenciamento dos Resíduos de Serviço de Saúde (PGRSS), além de classificar os resíduos em grupos de acordo com sua natureza em: A (infectantes), B (químicos), C (rejeitos radioativos), D (comuns) e E (perfurocortantes). Em 2005, o Conselho Nacional de Meio Ambiente (CONAMA) definiu e classificou os RSS como feito anteriormente pela ANVISA, e estabeleceu orientações quanto ao tratamento e a disposição final adequada de acordo com as características de cada classe por meio da Resolução nº 358 (CONAMA, 2005).

Nesse contexto, cada classe de resíduos deve seguir um fluxo de gerenciamento sendo devidamente segregados, acondicionados e armazenados. A deficiência no cumprimento de todas essas etapas propostas pelas legislações pertinentes resulta no aumento de volume de resíduos, bem como no custo de seu tratamento (HOSSAIN et al., 2011). Estudos realizados em hospitais concluíram que as práticas que caracterizam o manejo adequado também influenciam na redução da ocorrência de acidentes de trabalho dos profissionais de saúde e da limpeza, quando os resíduos são armazenados em locais seguros, sem o risco de contato, favorecendo a saúde ocupacional (SCHNEIDER, 2004; DINIZ et al., 2013).

Deve-se mencionar ainda que os cuidados requeridos pelos RSS precisam ser alcançados por meio de etapas de planejamento que abrangem aspectos referentes à estrutura física das unidades de saúde, à disponibilidade de materiais de trabalho, além de profissionais 
capacitados acerca dos RSS, levando em consideração suas características quantitativas e qualitativas norteados pelo PGRSS, que deve ser elaborado e implantado por um responsável técnico (ANVISA, 2004).

Um dos fatores determinantes para a definição do tratamento adequado é a quantidade de resíduos, que pode variar conforme o potencial econômico da região onde está localizada a instituição médica e o nível de desenvolvimento do país, pois o poder de consumo influencia significativamente no montante de resíduos gerados. A Organização Mundial de Saúde (WHO, 2014) destaca que os dados quantitativos e qualitativos de geração de resíduos são essenciais para fins de planejamento, além de subsidiar a melhoria dos processos dentro das unidades de saúde, conforme pode ser observado em diversas pesquisas em âmbito internacional (DEBERE et al., 2013; SANIDA et al., 2010; ZAZOULI et al., 2015; BAMBARÉN-ALATRISTA \& ALATRISTA-GUTIÉRREZ, 2014).

A gestão no ambiente hospitalar também reflete na quantidade e qualidade dos resíduos, e sua deficiência durante a segregação na fonte geradora acarreta o seu aumento devido à mistura de resíduos recicláveis aos resíduos contaminados, inviabilizando a parcela que poderia ser reaproveitada (CHENG et al., 2009). Para isso, deve-se ter uma visão holística da gestão, pois os procedimentos a serem seguidos para o gerenciamento adequado não devem concentrar-se apenas no manejo dos RSS e especificamente nas unidades geradoras, existindo assim uma dinâmica que envolve aspectos financeiros, políticos e educacionais, refletindo na qualidade ambiental do município (DINIZ et al., 2013). No Brasil, apesar das legislações existentes, ainda é necessário discutir sobre o uso e a viabilidade de novas tecnologias para o tratamento e a disposição final, que sejam acessíveis às diversas realidades encontradas no país. Deve-se levar em consideração que para alcançar a conformidade são necessários investimentos de or- dem financeira e científica, além de capacitação para os envolvidos no processo, visando à proteção dos trabalhadores, bem como a saúde ambiental (SILVA et al., 2011; GARCIA \& ZANETTI-RAMOS, 2004).

Outra questão que influi diretamente na gestão é a falta de conhecimento dos profissionais quanto à importância da totalidade das ações do gerenciamento. Em pesquisa realizada com 29 profissionais responsáveis pelos resíduos de diferentes unidades de saúde, Gessner et al. (2013) constatou-se que muitos dos entrevistados não souberam responder como era feita a coleta dos RSS na unidade, assim como o adequado tratamento e destinação final para os resíduos das classes A, B e E. Esse fato evidencia a realidade encontrada em várias unidades de saúde, em que há falta de investimentos na área de capacitação e treinamento dos profissionais, necessitando ações para que o gerenciamento dos resíduos seja reconhecido como parte integrante do trabalho a ser realizado.

Nos últimos anos tem crescido a preocupação com os RSS por meio da criação de legislações específicas que subsidiem sua gestão. Porém, em muitas prefeituras, apesar de terem instituído processos de coleta e tratamento nas unidades de saúde dos municípios, a preocupação com o manejo dentro das unidades de saúde ainda é deficitária, necessitando uma maior atenção por parte dos gestores e dos profissionais envolvidos (PEREIRA, 2012).

Diante do exposto, esta pesquisa teve por objetivo realizar um diagnóstico do gerenciamento dos RSS de unidades de saúde públicas municipais em 17 municípios do estado de Goiás. Os dados obtidos podem ser utilizados para subsidiar modelos de gestão adequados nas unidades geradoras, além de contribuir para a composição de banco de dados dos órgãos competentes, a fim de elaborar diretrizes e programas para buscar o manejo correto dos RSS, e favorecer a promoção da saúde da população e a qualidade ambiental dos municípios.

\section{MATERIAIS E MÉTODOS}

Realizou-se uma pesquisa com abordagem qualiquantitativa e descritiva observacional, que utilizou o emprego de formulários para o levantamento de informações com visitas em campo. Os formulários foram elaborados a fim de abranger os procedimentos gerenciais, operacionais e as características qualitativas e quantitativas dos RSS gerados nas unidades da rede pública de saúde em 17 municípios do estado de Goiás, e aplicados aos gestores ou responsáveis técnicos pelos resíduos gerados nas unidades visitadas, com o intuito de levantar informações sobre as etapas de segregação, acondicionamento, armazenamento interno e externo, transporte, tratamento e disposição final, baseado na Resolução CONAMA nº 358 (CONAMA, 2005) e na RDC 
$n^{0} 306$ (ANVISA, 2004). Foram abordadas também, perguntas referentes ao conhecimento e treinamento dos funcionários. $\mathrm{O}$ formulário utilizado no presente trabaIho foi submetido ao Comitê de Ética em pesquisa da Universidade Federal de Goiás, sendo aprovado conforme o Parecer 391.907/2013, sendo composto de 16 questões, descritas a seguir:

1. As unidades de saúde possuem o Plano de Gerenciamento de Resíduos do Serviço de Saúde (PGRSS)? ( ) SIM ( ) NÃO

2. Em caso afirmativo, qual profissional/empresa foi responsável pela confecção do PGRSS?

3. Há a separação dos resíduos por classes? ( ) SIM ( ) NÃO

4. Quais são as classes de resíduos gerados nas unidades de saúde? ( ) A ( ) B ( ) C ( ) D ( ) E

5. É realizado algum tipo de aproveitamento ou beneficiamento dos resíduos da classe $D$, nas unidades de saúde? ( ) SIM ( ) NÃO

6. Os resíduos da classe $D$ são destinados à coleta seletiva? ( ) SIM ( ) NÃO

7. Qual a quantidade de resíduos gerados por classe $A$, $B, C$, D e E? ( $\mathrm{kg} / \mathrm{mês}, \mathrm{kg} / \mathrm{semana})$ ?

8. Houve treinamento para os funcionários em relação à classificação, segregação e acondicionamento correto dos resíduos? ( ) SIM ( ) NÃO

9. Os funcionários responsáveis pelo manejo dos RSS fazem uso de equipamentos de proteção (individuais e coletivos)? ( ) SIM ( ) NÃO

10. Forma de armazenamento externo, o local possui quais características: ( ) Abrigo coberto e impermeabilizado; ( ) Exclusivo; ( ) Identificado; ( ) Não há abrigo

11. Frequência de coleta dos resíduos? ( ) semanal; ( ) mensal; ( ) quinzenal;outra/qual?

12. Responsável pela coleta de RSS nas unidades de saúde? ( ) Prefeitura; ( ) Empresa Terceirizada. Se empresa, qual o nome?

13.É realizado algum tipo de tratamento dos resíduos nas unidades de saúde? ( ) Autoclave; ( ) Queima a céu aberto; ( ) Micro-ondas; ( ) Outra; ( ) Nenhuma
14.Qual tratamento/destinação final é realizado pela empresa responsável pela coleta dos resíduos? ( ) Incineração; ( ) Autoclavagem; ( ) Queima a céu aberto; ( ) Micro-ondas; ( ) Disposição em aterro; ( ) Lixão; ( ) outra. Qual?

15.É cobrado pela empresa algum valor pelo tratamento dos resíduos ( $\mathrm{Kg}$ )? ( ) Sim, quanto?; ( ) Não

16.Qual é o veículo utilizado para coleta dos resíduos? ( ) Veículo destinado a coletar exclusivamente lixo das unidades de saúde hospitalares; ( ) Veículo destinado a coletar o lixo comum; ( ) Outro/qual?

Para a realização do estudo foram pesquisadas 142 unidades de saúde públicas incluindo postos e unidades básicas de saúde da família, hospitais, clínicas odontológicas e secretarias de saúde do âmbito municipal (BRASIL, 2010) nos 17 municípios do estudo, que representam 6,91\% dos 246 municípios goianos, englobando $4,03 \%$ da população (259.701 habitantes) do estado, que possui 6.434 .048 habitantes, segundo dados do Instituto Brasileiro de Geografia e Estatística (IBGE, 2010).

A escolha dos municípios foi realizada em função da distribuição espacial pelo estado de Goiás, que permite avaliar todas as suas regiões, e pela variação populacional de cada município, aliados ao fato dos serviços de água e esgoto não serem operados pela Companhia Saneamento de Goiás (SANEAGO) e sim pela prefeitura local por meio de autarquias, secretarias ou sem nenhuma gestão. Esses municípios estão destacados na Figura 1, juntamente com as quantidades existentes de unidades de saúde públicas, objeto de estudo da pesquisa. A área de estudo inicial foi composta por 21 municípios, porém, em 1 não foi possível marcar a visita e 3 deles não aceitaram participar e não puderam ser incluídos nesta pesquisa conforme as exigências do Comitê de Ética da UFG, embasado pela Resolução CNS n 196/1996 que contém as diretrizes e normas regulamentadoras de pesquisas envolvendo seres humanos.

A coleta de dados foi iniciada em fevereiro de 2013 e dividida em três etapas:

1. contato telefônico e apresentação do projeto ao gestor responsável pelos RSS do município; 


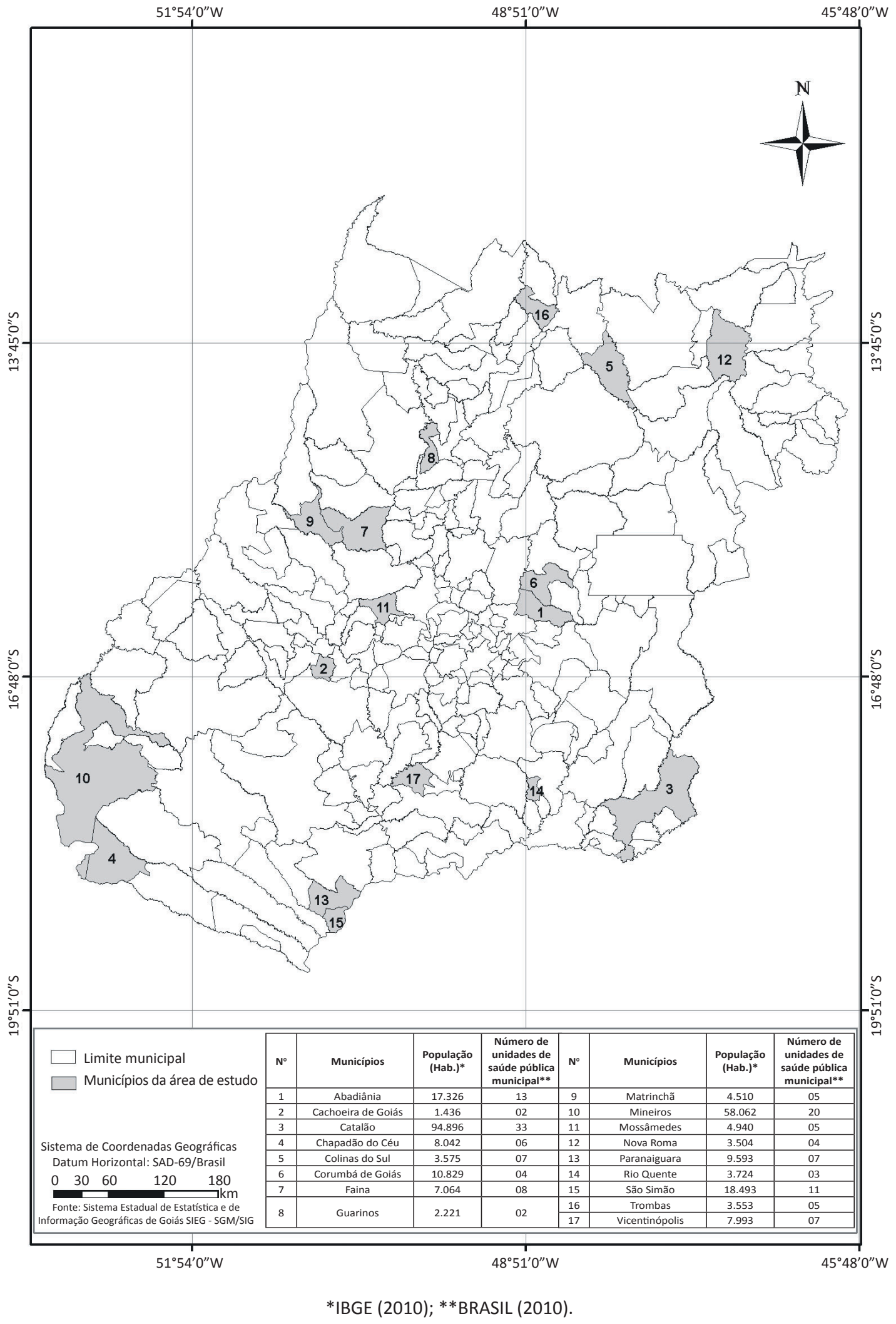

Figura 1 - Estado de Goiás com a distribuição espacial dos 17 municípios integrantes da pesquisa e a quantidade existente de unidades públicas de saúde. 
2. aplicação do formulário aos gestores; e

3. observação direta dos procedimentos referentes aos resíduos nas unidades de saúde, verificando as etapas do gerenciamento que são realizadas no interior das mesmas.
Os dados coletados foram subdivididos e relacionados com as diferentes etapas do gerenciamento, com o objetivo de avaliá-los conforme legislação pertinente. Para isso, utilizou-se como recurso metodológico o software Excel para inserção dos dados por meio de planilhas eletrônicas.

\section{RESULTADOS E DISCUSSÃO}

Nos municípios estudados, verificou-se que há três formas de estruturação dos serviços de coleta, tratamento e disposição final. Em 13 municípios analisados (76,47\%) esses serviços são realizados por empresa privada contratada pela prefeitura. Nota-se ainda que em 3 municípios (17,64\%), a prefeitura compartilha a realização desses serviços com uma empresa contratada e, em apenas 1 município (5,89\%), a prefeitura gerencia todas as etapas.

De acordo com a ANVISA (2004), os geradores de RSS devem elaborar o PGRSS, que deve conter todas as etapas do gerenciamento, a fim de obter um diagnóstico das características quantitativas e qualitativas dos resíduos gerados nas unidades de saúde para subsidiar a definição de ações para o alcance do manejo eficiente, visando à diminuição da produção dos resíduos gerados, assim como o seu encaminhamento para o tratamento e a disposição adequados. Essas ações refletem na manutenção da saúde pública e ambiental, bem como na proteção dos trabalhadores, sendo essencial a revisão periódica e o acompanhamento do plano. Constatou-se que as secretarias da saúde de 8 municípios $(47,05 \%)$ possuem o PGRSS, sendo que em 6 desses municípios (75\%) os planos foram elaborados por profissionais da área de enfermagem pertencentes ao quadro funcional da própria secretaria municipal, e nos 2 municípios restantes (25\%), por empresas contratadas.

Com relação a cursos de capacitações direcionados aos profissionais envolvidos no processo, observou-se que em 6 municípios $(35,29 \%)$ não eram oferecidos tais cursos, limitando o conhecimento dos trabalhadores ao que foi recebido em sua formação profissional. Dos 9 municípios que não possuem o PGRSS, apenas 4 relataram não oferecer cursos de capacitações, enquanto dos 8 municípios que possuem o plano, 2 deles (25\%) não o cumpriam quanto à promoção das capacitações, evidenciando a falta de comprometimento com o documento.
A realização de cursos de capacitação profissional reflete diretamente no gerenciamento dos RSS, uma vez que o conhecimento quanto aos procedimentos adequados a serem utilizados dentro das unidades é importante para uma segregação efetiva e consequente redução. Oli et al. (2016) verificaram em sua pesquisa, com 660 questionários aplicados em hospitais públicos e privados do sudeste da Nigéria, que apenas 71 profissionais haviam participado de algum treinamento referente ao gerenciamento de RSS, corroborando a alta geração de resíduos dos hospitais, resultado da deficiência na segregação e falta de conhecimento dos profissionais de saúde. Araújo et al. (2013), Narendra et al. (2013) e Gessner et al. (2013) também obtiveram resultados semelhantes quanto ao conhecimento relacionado ao gerenciamento dos RSS pelos profissionais de saúde.

Em todos os municípios estudados nesta pesquisa, os funcionários entrevistados relataram a interferência da mudança de gestão municipal na organização do estabelecimento de saúde, influenciando não somente na elaboração do PGRSS nos municípios que não o possuem, mas também na efetivação dos planos dos municípios que já possuíam tal plano.

Dentre as soluções para redução dos problemas relacionados com os RSS, a minimização de sua produção é uma das melhores opções, o que se torna difícil em decorrência do aumento progressivo do uso de materiais descartáveis, da dificuldade na manipulação de alguns resíduos devido à sua carga infecciosa e à reduzida oferta de cursos de capacitação. Portanto, a segregação correta no momento da geração é a solução mais apropriada e deve ser realizada por profissionais conscientes acerca dos problemas que envolvem o manejo dos RSS, resultando em uma gestão eficiente (CIPLAK \& BARTON, 2012).

Em estudo realizado por Araujo et al. (2013) em um hospital público no estado do Piauí, que avaliou o co- 
nhecimento dos enfermeiros sobre as etapas de gerenciamento dos RSS, foi verificado que $29,2 \%$ dos entrevistados não detinham conhecimento suficiente sobre esses resíduos, resultando no descarte incorreto, podendo comprometer a segregação dos resíduos na unidade de saúde. Da mesma forma, Narendra et al. (2013) constataram a falta de conhecimento referente às legislações pertinentes aos RSS, mesmo pelos profissionais mais capacitados em um hospital em Mysore, na Índia, concluindo sobre a importância da educação para o aprimoramento das ações relacionadas ao manejo de RSS.

Outro fator importante relacionado aos trabalhadores da limpeza nas unidades de saúde é quanto ao uso dos equipamentos de proteção individual (EPI), sendo observado que em apenas 1 município pesquisado $(5,88 \%)$ os responsáveis pela higienização da unidade de saúde não faziam uso de EPI. Nos demais municípios os profissionais utilizavam somente luvas e botas, fato esse também apontado por Pereira et al. (2010), comprometendo a proteção do trabalhador em sua totalidade, principalmente nos casos em que o gerenciamento dos resíduos não é realizado de maneira adequada, aumentando ainda mais os riscos.

Quanto à segregação dos resíduos, todos os gestores afirmaram realizar a separação de acordo com as classes, porém em 5 municípios $(29,41 \%)$ foi observado o improviso dos recipientes de acondicionamento dos resíduos do grupo $\mathrm{E}$ (Figura 2), não obedecendo às normas estabelecidas pela ANVISA (2004). Os recipientes improvisados não são próprios para esse tipo de acondicionamento, uma vez que não foram submetidos a testes que comprovem sua eficiência quanto à rigidez e resistência à punctura, ruptura e vazamento como estabelecido pela NBR 13853 (ABNT, 1997), além de ausência de identificação.

Em 5 municípios (29,41\%) estudados foram encontradas irregularidades com relação ao acondicionamento dos resíduos do grupo A (Figura 3), uma vez que, na falta de sacos brancos, são utilizados sacos pretos e azuis, entrando em divergência com a legislação vigente. Esse tipo de problema também foi encontrado em estudos realizados em três hospitais do estado de Mato Grosso do Sul onde os resíduos eram acondicionados em recipientes impróprios que extrapolavam o limite da capacidade permitida pela RDC $n^{0} 306$ (ANVISA, 2004), sendo também os sacos frágeis, maximizando os riscos aos trabalhadores e ao meio ambiente no momento do transporte interno e externo (TIVIROLLI et al., 2010). Esse fato também foi constatado por Pereira et al. (2010) em um hospital público da cidade de Campina Grande, na Paraíba. A justificativa encontrada na presente pesquisa para esse problema, relatada pelos entrevistados, foi a dificuldade na compra desses materiais, que depende de verba da prefeitura, prejudicada pela burocracia envolvida no processo de compra.

Em relação aos resíduos recicláveis pertencentes ao grupo D, verificou-se que não é realizado o encaminhamento para reciclagem em nenhum dos municípios estudados, mesmo naqueles que ofereciam o programa
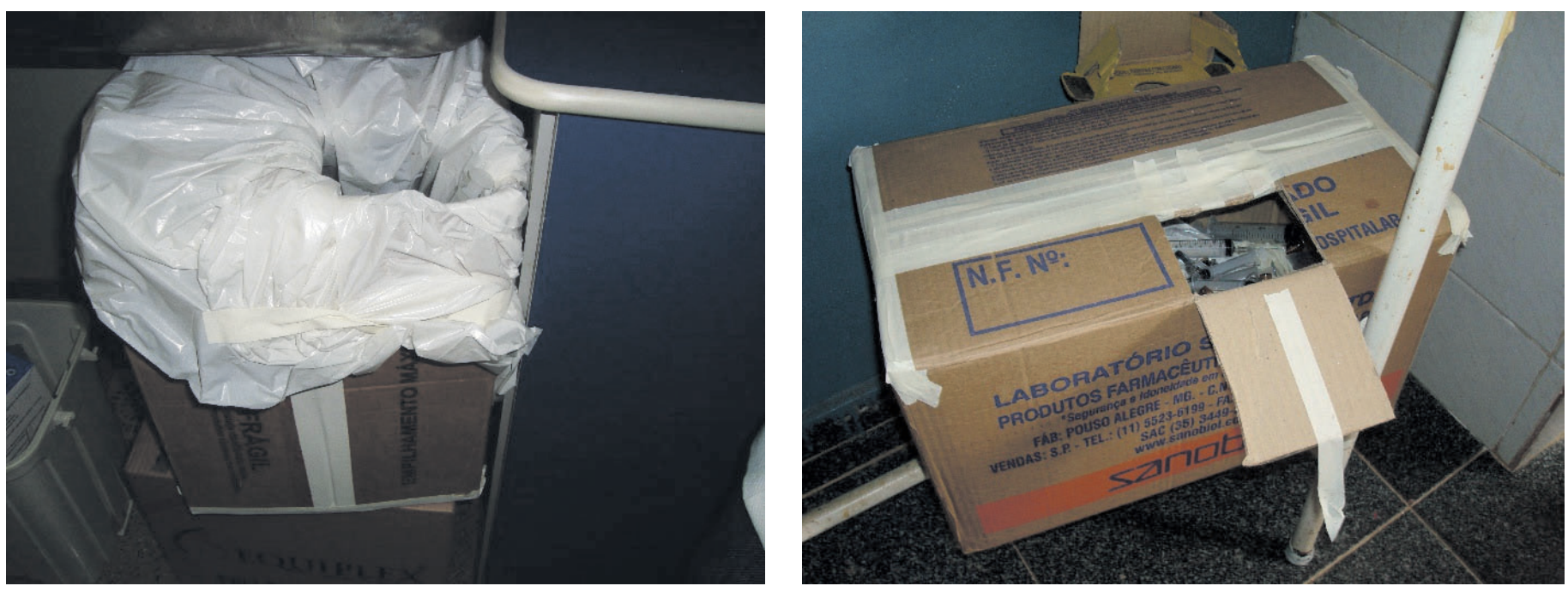

Figura 2 - Condições de improviso dos recipientes utilizados para acondicionamento dos resíduos da classe E. 
de coleta seletiva, inviabilizando a reciclagem daqueles que poderiam ser comercializados e servir como fonte de receita para o hospital, tais como embalagens de papelão e sacos plásticos, presentes em grande quantidade nos estabelecimentos de saúde estudados. Foi observada a mistura de resíduos da classe $D$ com a da classe $A$ em 5 municípios $(29,41 \%)$, o que dificulta uma possível reciclagem e reflete no aumento da quantidade de resíduos infectantes, que são encaminhados a um processo de tratamento, elevando os custos para a unidade de saúde. Situação semelhante foi verificada por Nicosia et al. (2013) em quatro departamentos de um hospital público em Palermo, na Itália, onde os resíduos da classe $\mathrm{D}$ são destinados ao mesmo saco, ou seja, não são segregados de acordo com sua classe. Destes, $65,7 \%$ eram passíveis de reciclagem, representando cerca de $34 \%$ do total produzido pela unidade, sendo imprescindível a implantação de programas de coleta seletiva, além de concluir que os resíduos infectantes representam a menor parcela da produção da unidade de saúde.

Schneider et al. (2013), em pesquisa realizada objetivando avaliar os custos com os RSS em um hospital escola no Rio Grande do Sul, enfatizam que a segregação inadequada, além de refletir em elevados custos para a unidade, também traz riscos aos profissionais de saúde, de higienização e da coleta interna e externa. Portanto, a sensibilização dos responsáveis pela gestão dos resíduos nos municípios e nas unidades de saúde é essencial para que a etapa de segregação seja vista com maior seriedade, oferecendo espaço físico e materiais necessários aos profissionais da saúde para o manejo adequado, além buscar a inserção de novas políticas públicas que beneficiem o cumprimento das legislações estabelecidas pelos órgãos responsáveis.

Moreira \& Günther (2016) avaliaram, no prazo de um ano, quatro unidades básicas de saúde, propondo o desenvolvimento de um instrumento facilitador a fim de caracterizar a geração dos RSS. Foi observado que o montante de resíduos não recicláveis, recicláveis e os contaminados, estava, respectivamente, em torno de 52 a $60 \%$, de 5 a $17 \%$ e de 31 a $42 \%$. Os autores concluíram que a falta de capacitação dos profissionais envolvidos no processo contribuiu para a segregação dos resíduos de forma deficiente. Segundo a Organização Mundial de Saúde (WHO, 2014), tipicamente a maior parcela de resíduos produzidos em ambientes hospitalares é de caráter não perigoso (75 a 90\%), enquanto os resíduos perigosos representam entre 10 e $25 \%$. Desse modo, é possível observar como o conhecimento dos profissionais de saúde quanto ao gerenciamento adequado dos RSS contribui significativamente para a quantidade de resíduos a serem tratados.

Durante a coleta de dados foram encontradas dificuldades no levantamento de informações referentes à quantificação dos RSS por classe, pois quando a quantificação era existente, apenas a quantidade total do resíduo coletado era contabilizada, ou seja, o somatório das classes A, B e E. Dos 17 municípios estudados, 2 não realizavam a pesagem e não estimavam a massa dos RSS gerada e, em outros 2 , o entrevistado não pos-
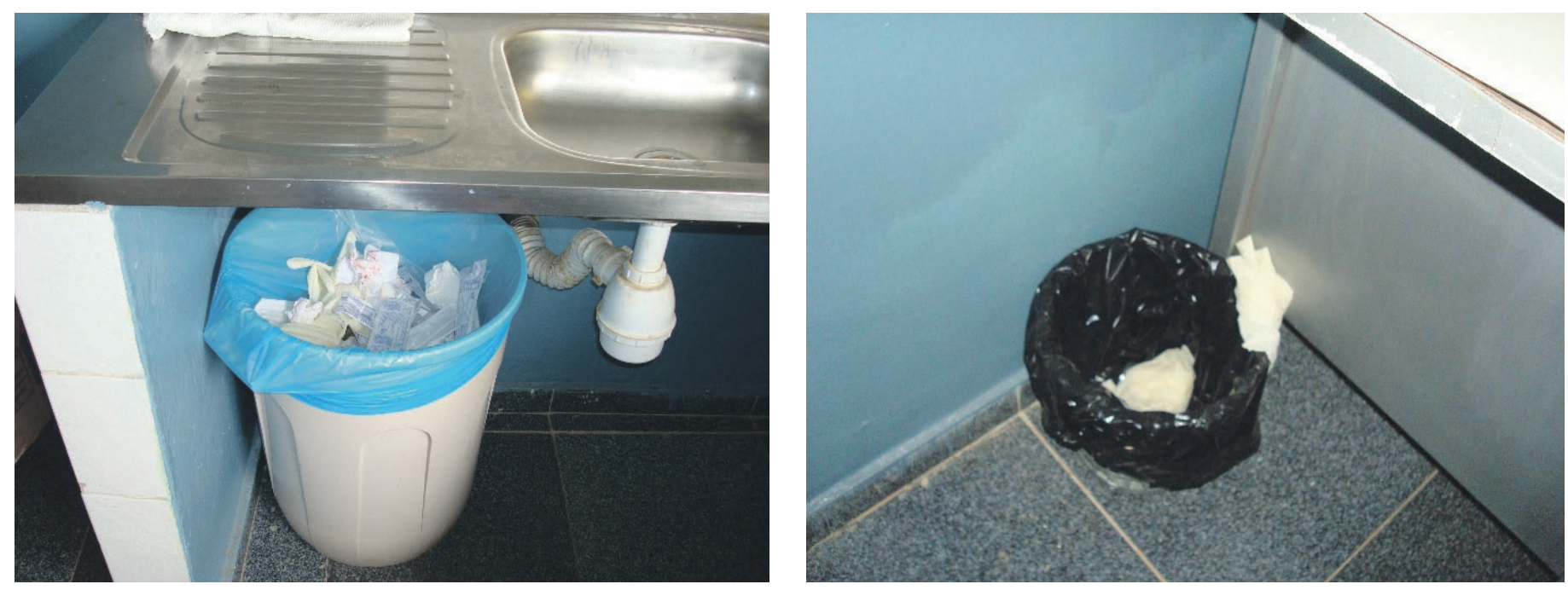

Figura 3 - Condições de improviso dos sacos utilizados para acondicionamento dos resíduos da classe A. 
suía tal informação. Os 13 municípios restantes apresentaram uma variação de geração de resíduos entre 0,02 e 1,26 kg.hab ${ }^{-1}$.ano ${ }^{-1}$, disparidade explicada pela variação populacional da área de estudo e pelos órgãos geradores de RSS atendidos. Essa variação pode ser observada na Figura 4, com destaque para o município 3 , que produz $1,26 \mathrm{~kg}$.hab ${ }^{-1}$.ano ${ }^{-1}$, e para o município 4 com $0,64 \mathrm{~kg} \cdot \mathrm{hab}^{-1}$.ano ${ }^{-1}$. Nesses dois municípios os serviços de coleta, tratamento e disposição final são de responsabilidade da prefeitura, que realiza a coleta nos estabelecimentos de saúde municipal e de todos os geradores de RSS do município. Esses estabelecimentos incluem farmácias, clínicas veterinárias, odontológicas e de estética, entre outros. A quantidade de resíduos gerados nos municípios 2, 6, 8, 11, 15 e 16 (Figura 4), é aquela estabelecida no contrato para sua retirada, ou seja, é um valor estimado mensalmente. Para a quanti- ficação da produção de RSS gerados diariamente, a forma mais comumente usada é a kg de resíduo por leito por dia $\left(\mathrm{kg}\right.$.leito $\left.{ }^{-1} \cdot \mathrm{d}^{-1}\right)$ e $\mathrm{kg}$ de resíduo por paciente por dia (kg.paciente $\mathrm{e}^{-1} \cdot \mathrm{d}^{-1}$ ), que deve ser definida de acordo com as informações fornecidas pelo prestador de serviço, bem como a diversidade das atividades existentes nas unidades de saúde. Essa forma de quantificação pode ser observada em diferentes estudos, tais como o de Debere et al. (2013), que realizaram o levantamento em 5 hospitais de Addis Ababa (Etiópia) e verificaram que a taxa de geração de RSS variou de 0,361 a 0,669 $\mathrm{kg}$. paciente ${ }^{-1} \cdot \mathrm{d}^{-1}$. Em um órgão da saúde na Macedônia (norte da Grécia) verificou-se que a geração dos RSS foi de 0,51 a 1,22 kg.paciente ${ }^{-1} \cdot \mathrm{d}^{-1}$ (SANIDA et al., 2010). $\mathrm{Na}$ presente pesquisa, devido à falta de informações da quantidade total de resíduos dos estabelecimentos visitados e da estruturação das unidades, foi utilizada a

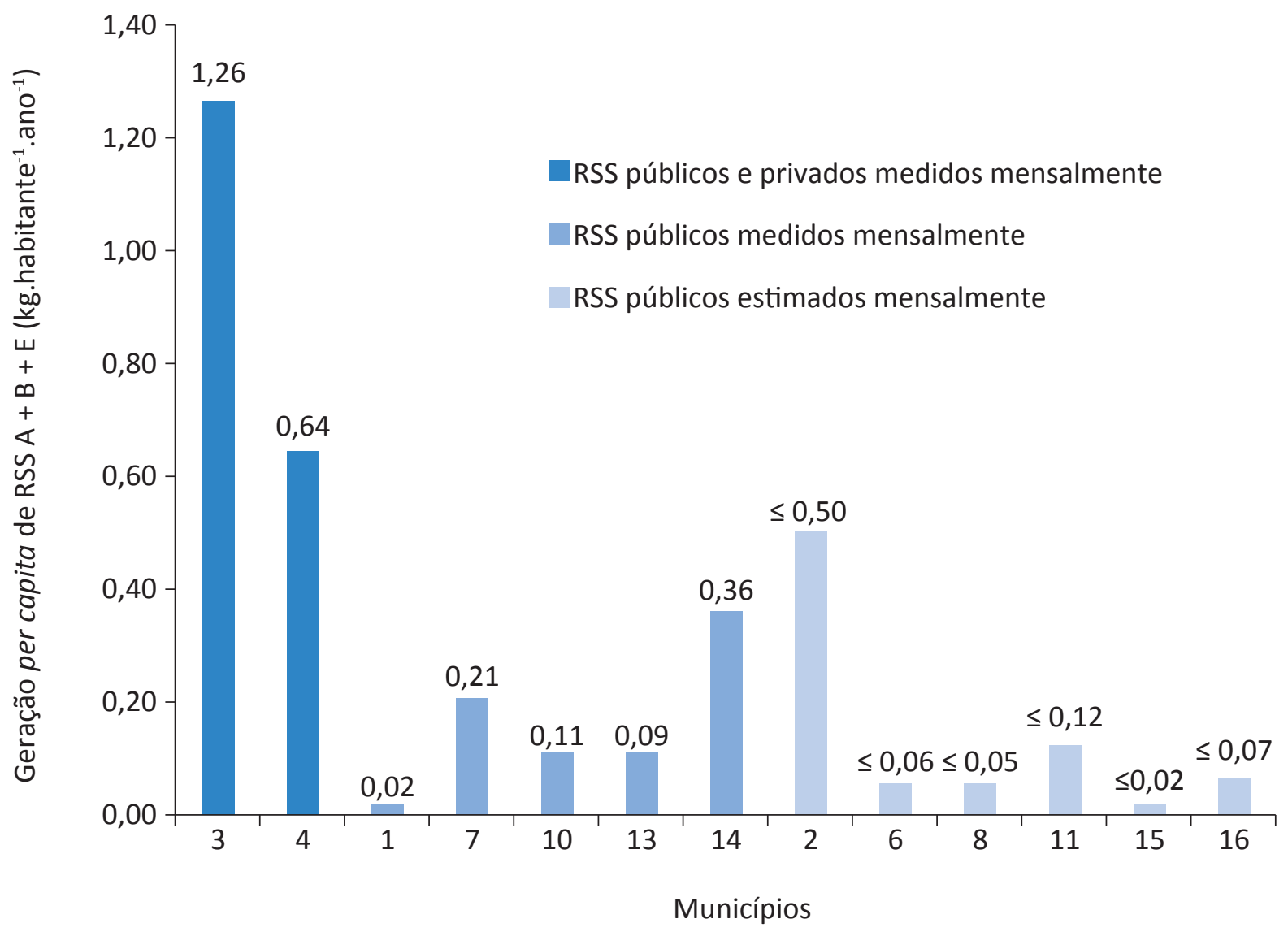

RSS: resíduos de serviços de saúde

Figura 4 - Geração de resíduos de serviços de saúde per capita em função das fontes geradoras (públicas e privadas) e formas de contabilização. 
geração per capita, que relaciona a quantidade de resíduos gerados com o número de habitantes do município estudado. De acordo com o Panorama de Resíduos Sólidos no Brasil referente ao ano de 2012, o estado de Goiás coletou cerca de 1,285 kg.hab ${ }^{-1}$.ano ${ }^{-1}$, apresentando uma quantidade maior quando comparado aos municípios da presente pesquisa (ABRELPE, 2012).

Quanto ao armazenamento externo, observa-se que em 2 municípios (11,76\%) não havia abrigo e os RSS ficavam em um espaço na área do hospital sem nenhuma estrutura de proteção, não sendo de acesso restrito, oferecendo riscos aos trabalhadores e à população. Foi observado também que $40 \%$ dos abrigos visitados não possuíam identificação sobre o armazenamento de resíduos infectantes.

Em nenhum dos municípios estudados foi encontrada a presença de abrigo exclusivo para os resíduos do grupo $B$, conforme estabelece a RDC $n^{\circ} 306$, sendo armazenados indevidamente juntamente com o grupo A e E. O armazenamento desse tipo de resíduo deve ainda seguir as orientações da NBR 12235 (ABNT, 1992) e a sua identificação, a NBR 7500 (ABNT, 2009).

Em apenas 3 municípios $(17,64 \%)$ os sacos contendo os resíduos eram armazenados em recipientes adequados, como preconiza a RDC $n^{\circ} 306$ (ANVISA, 2004), que foram fornecidos pela empresa prestadora de serviço. Já em relação ao tratamento prévio dos resíduos dentro das unidades de saúde antes da coleta interna, apenas 1 município $(5,88 \%)$ faz uso do processo de autoclavagem, como estabelece a Resolução CONAMA $n^{\circ}$ 358 (CONAMA, 2005), acarretando riscos aos trabalhadores e ao meio ambiente.

Em todos os municípios utiliza-se a incineração como método de tratamento total ou parcial. Em 2 deles $(11,76 \%)$ esse serviço é prestado pela própria prefeitura e no restante $(88,23 \%)$ é realizado por empresa contratada. Nos municípios onde o incinerador é operado pela prefeitura, ocorre a queima de aproximadamente 10 toneladas.mês ${ }^{-1}$, que corresponde ao tratamento do RSS gerado por $23 \%$ da população da área de estudo. Dessa forma, o funcionamento correto dos equipamentos, tanto dos sistemas municipais quanto das empresas contratadas, é de extrema importância, visto que apesar da incineração reduzir significativamente o volume dos resíduos, libera uma grande variedade e quantidade de gases poluentes, tais como metais pesa- dos, gases ácidos, monóxido de carbono e óxido de nitrogênio, podendo causar impactos ao meio ambiente e consequentemente à saúde pública, se não tratados da forma adequada. Para isso é necessário o incentivo no desenvolvimento de novas tecnologias ambientalmente seguras para o tratamento dos RSS, levando em consideração características relacionadas ao custo e à acessibilidade (HOSSAIN et al., 2011; CIPLAK \& BARTON, 2012).

Em 2 municípios (11,76\%) os resíduos do grupo E são encaminhados devidamente segregados à empresa contratada que realiza os serviços de coleta, tratamento e disposição final utilizando a incineração como método de tratamento. Os resíduos restantes, incluindo os grupos A, B e D, são coletados pelas prefeituras utilizando os mesmos profissionais e veículo de coleta dos RSU e, em seguida, dispostos em valas no lixão, onde são queimados e recobertos com solo. Essa situação desencadeia riscos, principalmente aos profissionais envolvidos nos processos de coleta interna e externa, sendo necessário o uso de EPIs específicos, os quais são preconizados pela NBR 12810 (ABNT, 1993). Nessa configuração, a disposição dos RSS diretamente no solo, sem qualquer tratamento, o contamina, podendo alcançar o lençol freático, comprometendo a saúde ambiental da região (GOMES, 2011). De acordo com os gestores desses dois municípios, o acúmulo dos resíduos do grupo A pode chegar a até dois meses, um período muito longo, o que contribui para a geração de um forte mau cheiro. Uma forma encontrada para tentar minimizar esse problema foi dispor esses resíduos no lixão, juntamente com os RSU do município. Esse período de tempo entre as coletas diverge do que preconiza a norma da NBR 12.810 (ABNT, 1993), na qual são estabelecidos que os intervalos máximos de coleta não devem ultrapassar 24 horas, podendo ser realizada em dias alternados, desde que os resíduos do grupo A estejam armazenados em um local com temperatura inferior a $4^{\circ} \mathrm{C}$. Verificou-se que as frequências de coleta quinzenal e mensal se destacaram com $41 \%$ cada uma (Figura 5), porém, somente 3 municípios $(17,6 \%)$ dispunham de refrigerador para o armazenamento desse resíduo.

Nos municípios onde ocorrem longos períodos de armazenamento, os principais motivos relatados pelos gestores estão relacionados ao difícil acesso à cidade, com vias sem pavimentação e estradas pre- 


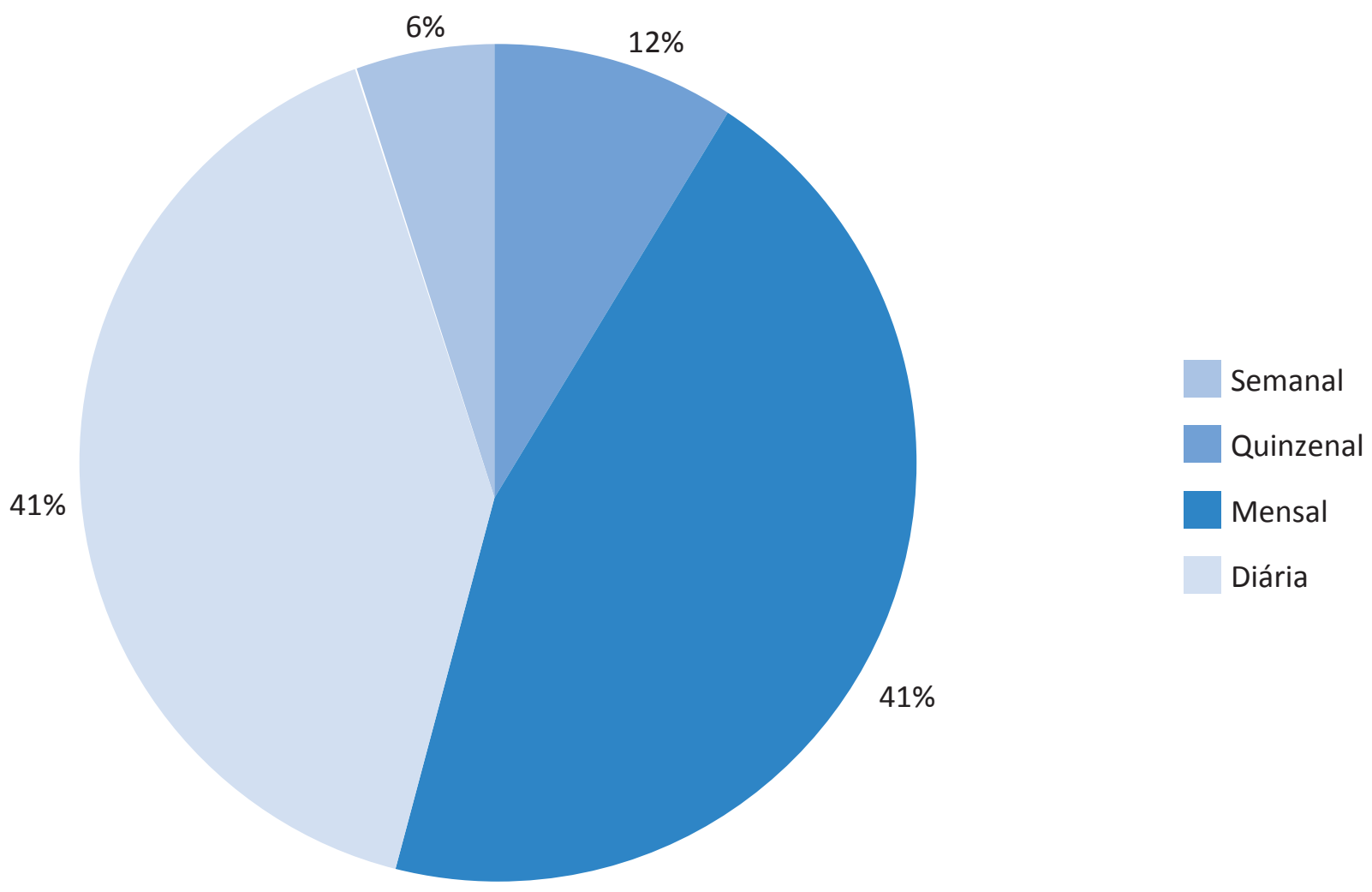

Figura 5 - Frequência de coleta dos resíduos de serviços de saúde.

cárias, e pela pequena quantidade de RSS produzida, prejudicando a relação custo-benefício para as empresas que realizam o trabalho. Nota-se que, nos municípios com menor contingente populacional, a frequência de coleta é menor em função da menor geração de RSS.

\section{CONCLUSÃO}

Apesar do conhecimento dos gestores sobre a obrigatoriedade da implantação do PGRSS e sua aplicação no cotidiano dos profissionais, constatou-se que apenas $47,05 \%$ dos municípios possuíam esse documento, o que resulta em falhas no cumprimento das etapas do gerenciamento em sua totalidade, refletindo no aumento de material contaminado e no descarte em local inapropriado.

As informações sobre a caracterização quantitativa e qualitativa dos RSS são deficientes, o que dificulta a completa avaliação. A obtenção desses dados é essencial para o planejamento das ações tanto no âmbito de capacitações dos profissionais quanto para diminuição dos custos. Desse modo, os gestores devem estabelecer rotinas nas unidades de saúde, a fim de promover esforços na segregação efetiva na fonte geradora, o qual deve estar em consonância com a implantação de programas de capacitações de forma contínua, buscando o aperfeiçoamento de técnicas seguras voltadas ao manejo adequado.

Em relação à disposição final, dois municípios ainda dispõem parte dos RSS em lixões, apesar do conhecimento sobre sua periculosidade e diante das leis existentes. Os gestores das unidades de saúde e o Poder Público devem promover o cumprimento das legislações existentes e o apoio na busca de novos métodos de tratamento, que sejam acessíveis a todos os municípios (incluindo os com dificuldade de acesso), além da disponibilização de recursos para infraestrutura e insumos, reconhecendo a gestão 
adequada dos RSS como ação indispensável para a manutenção da saúde coletiva.

A consolidação de procedimentos de gerenciamento dos RSS nas unidades de saúde é primordial, uma vez que nos municípios estudados foi encontrada uma forte intervenção política, influenciando no andamento dos processos de gestão dos resíduos.

O presente trabalho permitiu concluir que a gestão segura dos RSS é imprescindível para a qualidade do ambiente hospitalar, reduzindo riscos aos profissionais da saúde, dos pacientes e do meio ambiente. Nota-se uma tendência no aumento da geração de RSS devido ao uso cada vez maior de materiais descartáveis em decorrência da preocupação quanto à seguridade dos pacientes, porém, esse avanço deve ser acompanhado de ações cada vez mais pontuais no gerenciamento dos resíduos dentro das unidades de saúde, visando sua minimização por meio da segregação adequada, além do incentivo à reciclagem dos resíduos do grupo $D$, que são passíveis desse processo, o que contribui para a qualidade ambiental da localidade.

\title{
AGRADECIMENTOS
}

\begin{abstract}
À Fundação de Amparo a Pesquisa do Estado de Goiás (FAPEG) sob o Processo $n^{0} 201010267000775$, referente à

chamada pública 03/2010, e aos profissionais das unidades de saúde visitadas, que colaboraram com a pesquisa.
\end{abstract}

\section{REFERÊNCIAS}

AGÊNCIA NACIONAL DE VIGILÂNCIA SANITÁRIA (ANVISA). Resolução da Diretoria Colegiada n. ${ }^{\circ}$ 306, de 7 de dezembro de 2004. Dispõe sobre o Regulamento Técnico para o Gerenciamento de Resíduos de Serviços de Saúde. Diário Oficial da União, 10 dez. 2004.

ARAÚJO, R. I.; OLIVEIRA, E. A. R.; FORMIGA, L. H. F.; FORMIGA, L. M. F.; DE BRITO, B. B. Conhecimento do enfermeiro acerca do destino dos resíduos hospitalares. Revista Multiprofissional em Saúde do Hospital São Marcos, v. 1, n. 1, p. 31-39, 2013. Disponível em: <http://www.repositorio.ufc.br/bitstream/riufc/7945/1/2013_art_lholima2.pdf>. Acesso em: jun. 2014.

ASSOCIAÇÃO BRASILEIRA DE EMPRESAS DE LIMPEZA PÚBLICA E RESÍDUOS ESPECIAIS (ABRELPE). Panorama de Resíduos Sólidos no Brasil. 2012.

ASSOCIAÇÃO BRASILEIRA DE NORMAS TÉCNICAS (ABNT). NBR 7500: identificação para o transporte terrestre, manuseio, movimentação e armazenamento de produtos. Rio de Janeiro: ABNT, 2009.

. NBR 12235: armazenamento de resíduos sólidos perigosos. São Paulo: ABNT, 1992.

. NBR 12810: coleta de resíduos de serviços de saúde: procedimento. Rio de Janeiro: ABNT, 1993.

. NBR 13853: coletores para resíduos de serviços de saúde perfurantes ou cortantes: requisitos e métodos de ensaio. Rio de Janeiro: ABNT, 1997.

BAMBARÉN-ALATRISTA, C.; ALATRISTA-GUTIÉRREZ, M. S. Impacto ambiental de un hospital público en la ciudad de Lima, Perú. Revista Peruana de Medicina Experimental y Salud Publica, v. 31, n. 4, p. 712-715, 2014. Disponível em: <http://www.scielo.org.pe/pdf/rins/v31n4/a15v31n4.pdf>. Acesso em: dez. 2016.

BRASIL. Ministério da Saúde. Cadastro Nacional de Estabelecimentos de Saúde (CNES). 2010. Disponível em: <http:// www2.datasus.gov.br/DATASUS/index.php?area=02>. Acesso em: jul. 2013.

CHENG, Y. W.; SUNG, F. C.; YANG, Y.; LO, Y. H.; CHUNG, Y. T.; LI, K. C. Medical waste production at hospitals and associated factors. Waste Management, v. 29, p. 440-444, 2009. Disponível em:<http://dx.doi.org/10.1016/j.wasman.2008.01.014>. Acesso em: jul. 2013. 
CIPLAK, N.; BARTON, J. R. A system dynamics approach for healthcare waste management: a case study in Istanbul Metropolitan City, Turkey. Waste Management \& Research, v. 30, n. 6, p. 576-586, 2012. Disponível em: <http://dx.doi. org/10.1177/0734242X12443405>. Acesso em: jul. 2013.

CONSELHO NACIONAL DO MEIO AMBIENTE (CONAMA). Resolução n. ${ }^{\circ} 358$, de 29 de abril de 2005. Dispõe sobre o tratamento e a disposição final dos resíduos de serviços de saúde, e dá outras providências. Diário Oficial da União, 4 maio 2005.

DEBERE, M. K.; GELAYE, K. A.; ALAMDO, A. G.; TRIFA, Z. M. Assessment of the health care waste generation rates and its management system in hospitals of Addis Ababa, Ethiopia. BMC Public Health, v. 13, n. 1, p. 1-9, 2013. Disponível em: <http://dx.doi:10.1186/1471-2458-13-28>. Acesso em: dez. 2016.

DINIZ, É. J. D. M.; AZEVEDO, G. L. D.; SILVA, C. R. A. D.; FERREIRA, C. M. F. Estudo observacional da gestão dos resíduos hospitalares em um hospital público da região do Trairi. Revista Bioterra, v. 13, n. 1, p. 89-94, 2013. Disponível em: <http://joaootavio.com.br/bioterra/workspace/uploads/artigos/680-2773-1-pb-53df91c221ba6.pdf>. Acesso em: maio 2014.

GARCIA, L. P.; ZANETTI-RAMOS, B. G. Gerenciamento dos resíduos de serviços de saúde: uma questão de biossegurança. Cadernos de Saúde Pública, v. 20, n. 3, p. 744-752, 2004. Disponível em: <http://dx.doi.org/10.1590/S0102311X2004000300011>. Acesso em: maio 2014.

GESSNER, R.; PIOSIADLO, L. C. M.; FONSECA, R. M. G. S.; LAROCCA, L. M. O manejo dos resíduos dos serviços de saúde: um problema a ser enfrentado. Cogitare Enfermagem, v. 18, n. 1, p. 117-123, 2013. Disponível em: <http://ojs.c3sl. ufpr.br/ojs2/index.php/cogitare/article/view/31316/20023>. Acesso em: mar. 2014.

GOMES, J. N. Condições ambientais e análise social dos moradores do entorno do lixão no município Benevides, estado do Pará. Caminhos de Geografia, v. 12, n. 37, p. 305-309, 2011. Disponível em: <http://www.seer.ufu.br/index.php/ caminhosdegeografia/article/view/16217/9116>. Acesso em: mar. 2014.

HOSSAIN, M. S.; SANTHANAM, A.; NIK NORULAINI, N. A.; OMAR, A. K. Clinical solid waste management practices and its impact on human health and environment - a review. Waste Management, v. 31, p. 754-766, 2011. Disponível em: <http://dx.doi.org/10.1016/j.wasman.2010.11.008>. Acesso em: jun. 2011.

INSTITUTO BRASILEIRO DE GEOGRAFIA E ESTATÍSTICA (IBGE). Série Cidades. 2010. Disponível em: <http://cidades.ibge. gov.br/xtras/home.php>. Acesso em: jun. 2012.

MOREIRA, A. M. M.; GÜNTHER, W. M. R. Gestión de residuos sólidos en las unidades básicas de salud: aplicación de instrumento facilitador. Revista Latino-Americana de Enfermagem, v. 24, 2016. Disponível em: <http://dx.doi. org/10.1590/1518-8345.0646.2768>. Acesso em: dez. 2016.

NARENDRA, M.; KOUSAR, H.; PUTTAIAH, E. T.; THIRUMALA, S. Assessment of Biomedical waste of various hospitals in Mysore City Karnataka, India. International Journal of Current Microbiology and Applied Sciences, v. 2, n. 3, p. 1-5, 2013. Disponível em: <http://www.ijcmas.com/Archives/vol-2-3/Madhu\%20Narendra\%20et\%20al.pdf>. Acesso em: dez. 2013.

NICOSIA, S.; LANZA, P. A.; PRESTIGIACOMO, S.; MANCUSO, R.; VIVIANI, G. Experimental sorting of municipal-like waste in the hospital "Civico", Palermo (IT). American Journal of Environmental Protection, v. 2, n. 2, p. 64-71, 2013. Disponível em: <https://iris.unipa.it/retrieve/handle/10447/91750/85450/10.11648.j.ajep.20130202.16.pdf>. Acesso em: dez. 2013.

OLI, A. N.; EKEJINDU, C. C.; ADJE, D. U.; EZEOBI, I.; EJIOFOR, O. S.; IBEH, C. C.; UBAJAKA, C. F. Healthcare waste management in selected government and private hospitals in Southeast Nigeria. Asian Pacific Journal of Tropical Biomedicine, v. 6, n. 1, p. 84-89, 2016. Disponível em: <http://dx.doi.org/10.1016/j.apjtb.2015.09.019>. Acesso em: dez. 2016. 
PÉPIN, J.; CHAKRA, C. N. A.; PÉPIN, E.; NAULT, V.; VALIQUETTE, L. Evolution of the global burden of viral infections from unsafe medical injections, 2000-2010. PLoS One, v. 9, n. 6, p. e99677, 2014. Disponível em: <https://www.ncbi.nlm. nih.gov/pmc/articles/PMC4049770/pdf/pone.0099677.pdf>. Acesso em: abr. 2017.

PEREIRA, S. S. Reflexões sobre o processo de urbanização e a necessidade de gestão ambiental: o caso dos resíduos de serviço de saúde da cidade de Campina Grande, PB. Reunir, v. 2, n. 1, p. 87-103, 2012. Disponível em: <http://150.165.111.246/revistaadmin/index.php/uacc/article/view/48>. Acesso em: dez. 2013.

PEREIRA, S. S.; LUCENA, L. L.; FERNANDES, A. Resíduos de serviço de saúde em um hospital de Campina Grande/PB: gestão e percepção ambiental. Revista Brasileira de Gestão e Desenvolvimento Regional, v. 6, n. 3, p. 255-286, 2010. Disponível em: <http://rbgdr.net/revista/index.php/rbgdr/article/view/322/220>. Acesso em: dez. 2013.

ROCHA, L. F. L. Análise comparativa das tecnologias empregadas para o tratamento de resíduos de serviços de saúde no Brasil. 43f. Trabalho de Conclusão de Curso (Bacharelado em Gestão Ambiental) - Faculdade de Planaltina, Universidade de Brasília, Planaltina, 2012. Disponível em: <http://bdm.unb.br/bitstream/10483/4086/1/2012_LuisFelipeLinoRocha. pdf>. Acesso em: mar. 2013.

SANIDA, G.; KARAGIANNIDIS, A.; MAVIDOU, F.; VARTZOPOULOS, D.; MOUSSIOPOULOS, N.; CHATZOPOULOS, S. Assessing generated quantities of infectious medical wastes: a case study for a health region administration in Central Macedonia, Greece. Waste Management, v. 30, n. 3, p. 532-538, 2010. Disponível em: <https://www.ncbi.nlm.nih.gov/ pubmed/19944583>. Acesso em: dez. 2016.

SCHNEIDER, V. E. Sistemas de gerenciamento de resíduos sólidos de serviços de saúde: contribuição ao estudo das variáveis que interferem no processo de implantação, monitoramento e custos decorrentes. 242f. Tese (Doutorado) Universidade Federal do Rio Grande do Sul, Porto Alegre, 2004. Disponível em: <http://www.lume.ufrgs.br/bitstream/ handle/10183/5982/000478819.pdf?sequence=1>. Acesso em: mar. 2013.

SCHNEIDER, V. E.; STEDILE, N. L. R.; BIGOLIN, M.; PAIZ, J. C. Sistema de Informações Gerenciais (SIG): Ferramenta de monitoramento do gerenciamento de resíduos de serviços de saúde (RSS) e dos Custos de tratamento. Revista de Gestão Ambiental e Sustentabilidade, v. 2, n. 1, p. 166-188, 2013. Disponível em: <http://dx.doi:10.5585/geas.v2i1.18>. Acesso em: jul. 2013.

SILVA, A. C. N.; CARVALHO, J. J. S.; LUÍS, A. S. B.; SILVA, A. C. R.; MOTA, E. L. A.; REIS, M. G. Regulamentação do tratamento de resíduos infectantes em serviços de saúde: uma revisão de literatura. Revista Brasileira de Ciências Ambientais, v. 22, n. 4, p. 28-37, 2011. Disponível em: <http://abes-dn.org.br/publicacoes/rbciamb/PDFs/22-06_Materia_4_final_ artigos287.pdf>. Acesso em: maio 2014.

TIVIROLLI, K.; TIVIROLLI, S. C.; LUZ, P. C.; FUJINO, L. B. V.; SHINZATO, M. P.; SKOWRONSKI, J.; GOMES, A. O.; VASCONCELOS, L. H. A.; HESS, S. C. Gerenciamento dos resíduos em três hospitais públicos do Mato Grosso do Sul, Brasil. Revista Brasileira em Promoção da Saúde, v. 23, n. 3, p. 213-220, 2010. Disponível em: <http://dx.doi:10.5020/18061230.2010. p221>. Acesso em: maio 2014.

WORLD HEALTH ORGANIZATION (WHO). Safe management of wastes from health-care activities. 2. ed. Geneva: World Health Organization, 2014. Disponível em: <http://www.searo.who.int/srilanka/documents/safe_management_of_ wastes_from_healthcare_activities.pdf>Acesso em: 24 nov. 2016.

ZAZOULI, M. A.; ALAVINIA, S. M.; BAY, A. Medical Waste Generation in Gorgan Hospitals. Journal of Mazandaran University of Medical Sciences, v. 25 n. 132, p. 309-313, 2015. Disponível em: <http://www.sid.ir/fa/VEWSSID/J_ pdf/600139413230.pdf>. Acesso em: dez. 2016. 